\title{
Proton-Pump Inhibitors and Hypomagnesaemia in Kidney Transplant Recipients
}

\author{
Rianne M. Douwes ${ }^{1}$, $\mathbb{C}^{\mathbb{D}}$, António W. Gomes-Neto ${ }^{1}$, Joëlle C. Schutten ${ }^{1}$, Else van den Berg ${ }^{1}$, \\ Martin H. de Borst ${ }^{1}{ }^{(1)}$, Stefan P. Berger ${ }^{1}$, Daan J. Touw ${ }^{2}{ }^{(0)}$, Eelko Hak ${ }^{3}$, Hans Blokzij1 ${ }^{4}{ }^{(}$, \\ Gerjan Navis ${ }^{1}$ and Stephan J. L. Bakker ${ }^{1}$ (i) \\ 1 Department of Internal Medicine, Division of Nephrology, University Medical Center Groningen, University \\ of Groningen, 9700 RB Groningen, The Netherlands; a.w.gomes.neto@umcg.nl (A.W.G.-N.); \\ j.c.schutten@umcg.nl (J.C.S); e.van.den.berg@umcg.nl (E.v.d.B.); m.h.de.borst@umcg.nl (M.H.d.B.); \\ s.p.berger@umcg.nl (S.P.B.); g.j.navis@umcg.nl (G.N.); s.j.l.bakker@umcg.nl (S.J.L.B.) \\ 2 Department of Clinical Pharmacy and Pharmacology, University Medical Center Groningen, University of \\ Groningen, 9700 RB Groningen, The Netherlands; d.j.touw@umcg.nl \\ 3 Unit PharmacoTherapy, -Epidemiology and -Economics, Groningen Research Institute of Pharmacy, \\ University of Groningen, 9713 AV Groningen, The Netherlands; e.hak@rug.nl \\ 4 Department of Gastroenterology and Hepatology, University Medical Center Groningen, University of \\ Groningen, 9700 RB Groningen, The Netherlands; h.blokzij@@umcg.nl \\ * Correspondence: r.m.douwes@umcg.nl; Tel.: +31-50-3612277
}

Received: 30 October 2019; Accepted: 4 December 2019; Published: 6 December 2019

\begin{abstract}
Proton-pump inhibitors (PPIs) are commonly used after kidney transplantation and there is rarely an incentive to discontinue treatment. In the general population, PPI use has been associated with hypomagnesaemia. We aimed to investigate whether PPI use is associated with plasma magnesium, 24-h urinary magnesium excretion and hypomagnesaemia, in kidney transplant recipients (KTR). Plasma magnesium and 24-h urinary magnesium excretion were measured in 686 stable outpatient KTR with a functioning allograft for $\geq 1$ year from the TransplantLines Food and Nutrition Biobank and Cohort-Study (NCT02811835). PPIs were used by 389 KTR (56.6\%). In multivariable linear regression analyses, PPI use was associated with lower plasma magnesium ( $\beta$ : $-0.02, P=0.02)$ and lower 24 -h urinary magnesium excretion $(\beta:-0.82, P<0.001)$. Moreover, PPI users had a higher risk of hypomagnesaemia (plasma magnesium $<0.70 \mathrm{mmol} / \mathrm{L}$ ), compared with non-users (Odds Ratio (OR): 2.12; 95\% confidence interval (CI) 1.43-3.15, $P<0.001$ ). This risk tended to be highest among KTR taking high PPI dosages ( $>20 \mathrm{mg}$ omeprazole Eq/day) and was independent of adjustment for potential confounders (OR: 2.46; 95\% CI 1.32-4.57, $P<0.005$ ). No interaction was observed between PPI use and the use of loop diuretics, thiazide diuretics, tacrolimus, or diabetes $\left(P_{\text {interaction }}>0.05\right)$. These results demonstrate that PPI use is independently associated with lower magnesium status and hypomagnesaemia in KTR. The concomitant decrease in urinary magnesium excretion indicates that this likely is the consequence of reduced intestinal magnesium absorption. Based on these results, it might be of benefit to monitor magnesium status periodically in KTR on chronic PPI therapy.
\end{abstract}

Keywords: proton-pump inhibitors; magnesium; hypomagnesaemia; kidney transplantation

\section{Introduction}

Proton-pump inhibitors (PPIs) are frequently used after kidney transplantation for their gastro- protective properties in the setting of immunosuppressive therapy, which usually includes glucocorticoids. Since their first introduction in the late 1980s, numerous case reports and observational studies have been published that associate PPI use with unfavorable clinical outcomes, including an 
increased risk of hypomagnesaemia [1-8]. Recently, this observation has been strengthened by a large population based cohort study which demonstrated a two times higher risk of hypomagnesaemia among subjects from the general populations on chronic PPI therapy compared to non-users [9].

Magnesium homeostasis depends mainly on the balance between intestinal $\mathrm{Mg}^{2+}$ uptake, storage and resorption from bones and urinary excretion of $\mathrm{Mg}^{2+}$ via the kidneys [10]. It is postulated that PPIs induce hypomagnesaemia through inhibition of $\mathrm{pH}$-dependent active magnesium absorption via transient receptor potential melastatin (TRPM) 6 and 7 channels in the intestine [11,12]. Moreover, increased renal magnesium retention has been observed in magnesium depleted subjects using chronic PPI therapy, indicating a defect in intestinal magnesium absorption or increased losses into the gastrointestinal tract, rather than renal magnesium wasting $[1,7,13]$.

Hypomagnesaemia is very common after kidney transplantation and it is generally thought to be a side effect of immunosuppressive therapy, especially of calcineurin inhibitors (CNI) which are known to induce renal magnesium wasting [14]. It has been shown that hypomagnesaemia is not only present in the immediate post transplantation period, but persists in about $20 \%$ of kidney transplant recipients (KTR) for many years after transplantation [15,16]. Importantly, hypomagnesaemia has been associated with onset of post-transplant diabetes mellitus (PTDM) in KTR $[17,18]$ and has also been associated with increased risk of cardiovascular morbidity $[19,20]$ and mortality [21] in the general population. Whether use of PPIs contributes to hypomagnesaemia in KTR has not been well established. To our knowledge only one cohort study investigated the association between PPI use and hypomagnesaemia in 512 KTR, with negative results [22]. Reasons for absence of an association were unclear, but may have included a low prevalence of PPI use of $20 \%$, which could have led to low statistical power of the study. Thus, whether PPI use negatively affects magnesium status after transplantation remains to be determined. We aimed to investigate whether PPI use is associated with magnesium status and hypomagnesaemia in a large single center cohort of stable outpatient KTR, in which plasma magnesium measurements were not part of routine clinical care but were assessed from samples that had been stored in a biobank.

\section{Methods}

\subsection{Study Design and Population}

This is a cross-sectional analysis using data from a previously described prospective cohort study registered at clinicaltrials.gov as 'TransplantLines Food and Nutrition Biobank and Cohort-study', NCT02811835 [23]. In summary, all adult KTR with a functioning graft beyond the first year after transplantation and without known or apparent systemic illnesses (i.e., malignancies other than cured skin cancer, opportunistic infections, overt congestive heart failure) who visited the outpatient clinic of the University Medical Center Groningen (UMCG) between November 2008 and March 2011, were asked to participate. A total of 707 out of the initially 817 invited KTR signed informed consent. We excluded KTR with missing biomaterial $(n=8)$, missing data on PPI dosage $(n=1)$, with on-demand PPI use $(n=3)$ or using magnesium supplements $(n=6)$ from statistical analyses, leaving 689 cases eligible for analysis. Study measurements were performed during a single study visit at the outpatient clinic. All study procedures were conducted in adherence with the Declaration of Helsinki and Declaration of Istanbul. The institutional review board of the UMCG approved the study protocol (METC 2008/186, approved on 17 September 2008).

\subsection{Exposure Definition}

PPI type and daily dosage were obtained from electronic patient records and are demonstrated in Table S1. KTR using any PPI on a daily basis during a period of at least 3 months prior to the study visit were defined as chronic PPI users as described previously [24]. To investigate a potential dose-response relationship, KTR were divided into three groups based on daily PPI dose defined in 
omeprazole equivalents: no PPI, low PPI dose ( $\leq 20 \mathrm{mg}$ omeprazole equivalents/day (Eq/day)) and high PPI dose ( $>20 \mathrm{mg}$ omeprazole Eq/day) [24,25].

\subsection{Assessement of Plasma and Urinary Magnesium}

Plasma magnesium was measured in samples containing lithium heparin, using a xylidyl blue method (Roche Modular analyzer, Roche Diagnostics, Mannheim, Germany). Urinary magnesium excretion was assessed in $24 \mathrm{~h}$-urine samples and measured on a MEGA clinical chemistry analyzer (Merck, Darmstadt, Germany). Hypomagnesaemia was defined as plasma magnesium $<0.70 \mathrm{mmol} / \mathrm{L}$.

\subsection{Assessment of Dietary Magnesium Intake}

Dietary magnesium intake was calculated using a validated semi quantitative food frequency questionnaire (FFQ) developed and updated at the Wageningen University, which was filled out at home $[26,27]$. Dietary data were converted into daily nutrient intake using the Dutch Food Composition Table of 2006 [28].

\subsection{Assessment of Covariates}

Medical history was obtained from electronic patient records as described previously [23]. History of cardiovascular disease was classified according to the International Classification of Diseases, 10th revision (ICD-10) code Z86.7. Body mass index (BMI) was calculated as weight in kilograms divided by height in meters squared. Blood pressure was measured as described in detail previously [29]. Information on alcohol use and smoking behavior was obtained using a questionnaire. Medication use, including the use of PPIs, H2-receptor antagonists, diuretics, prednisolone, mycophenolate mofetil (MMF), tacrolimus, cyclosporine, and sirolimus was recorded at baseline. Routine immunosuppressive therapy consisted of: A combination of azathioprine and prednisolone from 1968 to 1989; a combination of cyclosporine and prednisolone from 1989 to 1996. In 1997 mycophenolate motefil was added to the standard immunosuppressive regimen and cyclosporine was slowly withdrawn after the first year in KTR without complications. In 2012 cyclosporine was replaced by tacrolimus, and KTR received triple-immunosuppressive therapy with prednisolone, tacrolimus and mycophenolate mofetil. PPIs were routinely prescribed after kidney transplantation for their gastro-protective properties with concurrent use of prednisolone. Blood samples were collected after an 8-12 h fasting period. Serum creatinine was measured using an enzymatic, isotope dilution mass spectrometry-traceable assay (P-Modular automated analyzer, Roche Diagnostics, Mannheim, Germany). Estimated glomerular filtration rate (eGFR) was calculated using the serum creatinine based Chronic Kidney Disease Epidemiology Collaboration (CKD-EPI) equation. Serum potassium, calcium, parathyroid hormone $(\mathrm{PTH})$, glucose and hemoglobin $\mathrm{A} 1 \mathrm{c}(\mathrm{HbA} 1 \mathrm{c})$, were determined using standard laboratory methods. Proteinuria was defined as urinary protein excretion $\geq 0.5 \mathrm{~g} / 24 \mathrm{~h}$.

\subsection{Statistical Analyses}

Statistical analyses were performed using SPSS, version 23.0 (IBM corp., Armonk, NY, USA). Data are presented as mean \pm SD for normally distributed data, median with interquartile range (IQR) for skewed data and number with percentage for nominal data. Differences between PPI users versus PPI non-users were tested using independent sample T-tests, Mann-Whitney U-tests and Chi-square tests or Fishers exact tests when appropriate.

To study the effect of PPI use on plasma magnesium linear regression analyses were performed with adjustment for potential confounders of magnesium status including: age, sex, BMI, eGFR, proteinuria, time since transplantation, alcohol use, diabetes, history of cardiovascular disease, use of loop diuretics, thiazide diuretics, tacrolimus, cyclosporine, MMF, and dietary magnesium intake. To investigate the association between PPI use and hypomagnesaemia we performed logistic regression analyses with adjustment for the same potential confounders used in multivariable linear regression analyses. Effect modification by loop diuretics, thiazide diuretics, tacrolimus and diabetes was tested 
by inclusion of interaction terms. To investigate a potential dose-response relationship we performed additional analyses in which KTR were divided into three groups based on daily PPI dose defined in omeprazole equivalents: No PPI, low PPI dose ( $\leq 20 \mathrm{mg}$ omeprazole Eq/day) and high PPI dose ( $>20 \mathrm{mg}$ omeprazole Eq/day) [24,25]. Tests of linear trend were conducted by assigning the median of daily PPI dose equivalents in subgroups treated as a continuous variable. We performed sensitivity analyses in which H2-receptor antagonist (H2RA) users $(n=18)$ were excluded to assess the robustness of the association between PPI use and hypomagnesaemia. Lastly, we investigated which KTR are at increased risk of developing hypomagnesaemia. A two-sided $P$-value $<0.05$ was considered statistically significant in all analyses.

\section{Results}

\subsection{Baseline Characteristics}

Baseline characteristics are shown in Table 1. PPIs were used by a small majority of $389(56.5 \%)$ KTR and omeprazole was the most often prescribed PPI $(n=340)$. Other PPIs used were esomeprazole $(n=30)$, pantoprazole $(n=16)$, and rabeprazole $(n=3)$. KTR who used PPIs were older than KTR who did not use PPIs, had a higher BMI and had shorter time between transplantation and baseline measurements. Diabetes was significantly more prevalent in PPI users compared with non-users $(28.3 \%$ vs. $18.3 \%, P<0.002)$. Plasma magnesium and 24 -h urinary magnesium excretion were significantly lower in PPI users and 102 (26.2\%) PPI users had hypomagnesaemia compared with $43(14.3 \%)$ non-users $(P<0.001)$. Dietary magnesium intake was not significantly different between PPI users and non-users. Loop diuretics, cyclosporine and MMF, were more often used by PPI users compared with non-users. Triple immunosuppressive therapy consisting of MMF, cyclosporine and prednisolone, was more common in PPI users compared with non-users. Duo therapy consisting of MMF-prednisolone, MMF-cyclosporine, and cyclosporine-prednisolone was more common in PPI users compared with non-users.

Table 1. Baseline characteristics of 689 kidney transplant recipients.

\begin{tabular}{|c|c|c|c|c|}
\hline Characteristics & Total Population & Non-PPI Users & PPI Users & $P$ \\
\hline Number of subjects, $n(\%)$ & $689(100)$ & $300(43.5)$ & $389(56.5)$ & $\mathrm{n} / \mathrm{a}$ \\
\hline \multicolumn{5}{|l|}{ Demographics } \\
\hline Age, year & $53 \pm 13$ & $51 \pm 13$ & $54 \pm 12$ & 0.001 \\
\hline Men, $n(\%)$ & $395(57.3)$ & $177(59.0)$ & $218(56.0)$ & 0.4 \\
\hline BMI, $\mathrm{kg} / \mathrm{m}^{2}$ & $26.6 \pm 4.8$ & $25.9 \pm 4.6$ & $27.1 \pm 4.8$ & 0.002 \\
\hline Diabetes Mellitus, $n(\%)$ & $165(23.9)$ & 55 (18.3) & $110(28.3)$ & 0.002 \\
\hline History of CV disease, $n(\%)$ & $274(39.8)$ & $92(30.7)$ & $182(46.8)$ & $<0.001$ \\
\hline Time since transplantation, year & $5.5(1.9-12.1)$ & $9.6(4.1-15.0)$ & $4.2(1.1-8.7)$ & $<0.001$ \\
\hline \multicolumn{5}{|l|}{ Lifestyle parameters } \\
\hline Current smoker, $n(\%)$ & $84(13.0)$ & $35(12.4)$ & 49 (13.6) & 0.7 \\
\hline Alcohol consumer, $n(\%)$ & $436(70.3)$ & $198(72.8)$ & $238(68.4)$ & 0.2 \\
\hline Magnesium intake, mg/day & $329.9 \pm 88.7$ & $333.0 \pm 89.2$ & $327.6 \pm 88.4$ & 0.5 \\
\hline \multicolumn{5}{|l|}{ Renal function parameters } \\
\hline $\mathrm{eGFR}, \mathrm{mL} / \mathrm{min} / 1.73 \mathrm{~m}^{2}$ & $52.3 \pm 20.2$ & $55.1 \pm 19.9$ & $50.2 \pm 20.1$ & 0.002 \\
\hline Serum creatinine, $\mu \mathrm{mol} / \mathrm{L}$ & $124(100-160)$ & $119(98-152)$ & $128(101-168)$ & 0.03 \\
\hline Proteinuria ( $\geq 0.5 \mathrm{~g} / 24 \mathrm{~h}), n(\%)$ & $157(22.9)$ & $71(23.7)$ & $86(22.2)$ & 0.7 \\
\hline \multicolumn{5}{|l|}{ Laboratory parameters } \\
\hline Hypomagnesaemia, $n(\%)$ & $145(21.0)$ & $43(14.3)$ & $102(26.2)$ & $<0.001$ \\
\hline Plasma magnesium, $\mathrm{mmol} / \mathrm{L}$ & $0.77 \pm 0.11$ & $0.79 \pm 0.09$ & $0.76 \pm 0.11$ & $<0.001$ \\
\hline 24-h urinary magnesium excretion, $\mathrm{mmol} / 24 \mathrm{~h}$ & $3.3(2.3-3.3)$ & $3.8(2.8-4.8)$ & $3.1(2.0-3.9)$ & $<0.001$ \\
\hline Serum potassium, $\mathrm{mmol} / \mathrm{L}$ & $3.98 \pm 0.46$ & $3.97 \pm 0.47$ & $3.99 \pm 0.46$ & 0.6 \\
\hline Serum calcium, mmol/L & $2.40 \pm 0.15$ & $2.40 \pm 0.15$ & $2.40 \pm 0.15$ & 0.8 \\
\hline $\mathrm{PTH}, \mathrm{pmol} / \mathrm{L}$ & $9.0(6.0-14.8)$ & $8.7(6.0-13.6)$ & $9.2(5.9-16.3)$ & 0.2 \\
\hline Glucose, mmol/L & $5.3(4.8-6)$ & $5.2(4.7-5.8)$ & $5.3(4.8-6.2)$ & 0.01 \\
\hline $\mathrm{HbA} 1 \mathrm{c}, \mathrm{mmol} / \mathrm{mol}$ & $40(37-44)$ & $39(36-42)$ & $41(38-45)$ & $<0.001$ \\
\hline
\end{tabular}


Table 1. Cont.

\begin{tabular}{|c|c|c|c|c|}
\hline Characteristics & Total Population & Non-PPI Users & PPI Users & $P$ \\
\hline \multicolumn{5}{|l|}{ Medication use } \\
\hline Mycophenolate mofetil, $n(\%)$ & $452(65.6)$ & $178(59.3)$ & $274(70.4)$ & 0.002 \\
\hline Tacrolimus, $n(\%)$ & $124(18.0)$ & $49(16.3)$ & 75 (19.3) & 0.3 \\
\hline Cyclosporine, $n(\%)$ & $272(39.5)$ & $97(32.3)$ & $175(45.0)$ & 0.001 \\
\hline Sirolimus, $n(\%)$ & $13(2.0)$ & $8(2.8)$ & $5(1.4)$ & 0.3 \\
\hline Prednisolone, $n(\%)$ & $682(99.0)$ & $298(99.3)$ & $384(98.7)$ & 0.7 \\
\hline Loop diuretics, $n(\%)$ & $160(23.2)$ & 41 (13.7) & 119 (30.6) & $<0.001$ \\
\hline Thiazide diuretics, $n(\%)$ & $120(17.4)$ & $53(17.7)$ & $67(17.4)$ & 0.9 \\
\hline H2-receptor antagonists, $n(\%)$ & $18(2.6)$ & $17(5.7)$ & $1(0.3)$ & $<0.001$ \\
\hline \multicolumn{5}{|l|}{ Combination therapy } \\
\hline MMF + Tac + pred, $n(\%)$ & $78(11.3)$ & $32(10.7)$ & $46(11.8)$ & 0.6 \\
\hline MMF + Cyclo + pred, $n(\%)$ & $175(25.4)$ & $51(17.0)$ & $124(31.9)$ & $<0.001$ \\
\hline $\mathrm{MMF}+\mathrm{Tac}, n(\%)$ & $81(11.8)$ & $33(11.0)$ & $48(12.3)$ & 0.6 \\
\hline MMF + Pred, $n(\%)$ & $447(64.9)$ & $176(58.7)$ & $271(69.7)$ & 0.003 \\
\hline MMF + Cyclo, $n(\%)$ & $177(25.7)$ & $52(17.3)$ & $125(32.1)$ & $<0.001$ \\
\hline Cyclo + Pred, $n(\%)$ & $269(39.0)$ & $96(32.0)$ & $173(44.5)$ & 0.001 \\
\hline Tac + Pred, $n(\%)$ & $120(17.4)$ & $48(16.0)$ & $72(18.5)$ & 0.4 \\
\hline
\end{tabular}

Data are presented as mean $\pm \mathrm{SD}$, median with interquartile ranges (IQR) or number with percentages $(\%)$. Abbreviations: BMI, body mass index; eGFR, estimated glomerular filtration rate; HbA1c, hemoglobin A1c; PTH, Parathyroid hormone; MMF, mycophenolate mofetil; Tac, tacrolimus; Pred, prednisolone.

\subsection{Association of PPI Use with Plasma Magnesium and 24-h urinary Magnesium Excretion}

PPI use was significantly associated with lower plasma magnesium $(\beta=-0.03 ; 95 \% \mathrm{CI}-0.04$; $-0.01 \mathrm{mmol} / \mathrm{L}, P=0.001)$ and lower urinary magnesium excretion $(\beta=-0.86 ; 95 \% \mathrm{CI}-1.10$; $-0.06 \mathrm{mmol} / 24 \mathrm{~h}, P<0.001)$ as compared to non-users, Table 2. After adjustment for potential confounders, PPI use remained significantly associated with lower plasma magnesium levels $(\beta=-0.02$, $95 \% \mathrm{CI}-0.04 ;-0.003, P=0.02)$ and 24 -h urinary magnesium excretion $(\beta=-0.82,95 \% \mathrm{CI}-1.07 ;-0.57$, $P<0.001)$.

Table 2. Association of proton-pump inhibitor (PPI) use with plasma magnesium and 24-h urinary magnesium excretion in 689 kidney transplant recipients.

\begin{tabular}{ccccccc}
\hline & \multicolumn{4}{c}{ Plasma Magnesium, mmol/L } & \multicolumn{4}{c}{ Urinary Magnesium Excretion, $\mathbf{m m o l} / \mathbf{2 4} \mathbf{~ h}$} \\
\cline { 2 - 7 } & $\boldsymbol{\beta}$ & $\mathbf{9 5 \%} \mathbf{C I}$ & $\boldsymbol{P}$ & $\boldsymbol{\beta}$ & $\mathbf{9 5 \%}$ CI & $\boldsymbol{P}$ \\
\hline Crude & -0.03 & $-0.04 ;-0.01$ & 0.001 & -0.86 & $-1.10 ;-0.06$ & $<0.001$ \\
Multivariable model & -0.02 & $-0.04 ;-0.003$ & 0.02 & -0.82 & $-1.07 ;-0.57$ & $<0.001$ \\
\hline
\end{tabular}

Multivariable analyses were adjusted for age, sex, BMI, eGFR, proteinuria, time since transplantation, alcohol use, diabetes, history of $\mathrm{CV}$ disease, loop diuretics, thiazide diuretics, tacrolimus use, cyclosporine use, MMF use and dietary magnesium intake. Abbreviations: CI, confidence interval.

\subsection{Association of PPI Use with Hypomagnesaemia}

In crude logistic regression analysis, PPI use was associated with a more than two times higher risk of hypomagnesaemia compared with no use (OR: 2.12; 95\% CI 1.43-3.15, $P<0.001$ ), as shown in Table 3. The association remained independent of adjustment for potential confounders including age, sex, eGFR, proteinuria, time since transplantation, alcohol use, diabetes, history of cardiovascular disease, medication use (loop diuretics, thiazide diuretics, tacrolimus, cyclosporine and MMF) and dietary magnesium intake (OR: 2.00; 95\% CI 1.21-3.31, $P=0.007$ ). No significant interaction was observed between PPI use and the use of loop diuretics, thiazide diuretics, tacrolimus, or diabetes for the association with hypomagnesaemia $\left(P_{\text {interaction }}=0.2, P_{\text {interaction }}=0.7, P_{\text {interaction }}=0.7, P_{\text {interaction }}=\right.$ 0.9 , respectively). 
Table 3. Logistic regression analyses investigating the association of PPI use with hypomagnesaemia in 689 kidney transplant recipients.

\begin{tabular}{cccc}
\hline & \multicolumn{3}{c}{ Hypomagnesaemia } \\
\hline $\mathbf{N}=\mathbf{6 8 9}$ & Odds Ratio & $\mathbf{9 5 \%}$ CI & $\boldsymbol{P}$ \\
\hline Crude & 2.12 & $1.43-3.15$ & $<0.001$ \\
Multivariable model & 2.00 & $1.21-3.31$ & 0.007 \\
\hline
\end{tabular}

Multivariable analyses were adjusted for age, sex, BMI, eGFR, proteinuria, time since transplantation, alcohol use, diabetes, history of $\mathrm{CV}$ disease, loop diuretics, thiazide diuretics, tacrolimus use, cyclosporine use, MMF use and dietary magnesium intake. Abbreviations: $\mathrm{CI}$, confidence interval.

\subsection{Dose-Response Analyses}

Based on daily dose equivalents of omeprazole, 251 KTR received a low PPI dose $(\leq 20 \mathrm{mg}$ omeprazole Eq/day) and $138 \mathrm{KTR}$ received a high PPI dose ( $>20 \mathrm{mg}$ omeprazole Eq/day). As shown in Table 4 and Figure 1, risk of hypomagnesaemia tended to be highest among KTR taking a high PPI dose (OR: 2.53; 95\% CI 1.55-4.11, $P<0.001$ ). The association remained materially unchanged after multivariable adjustment (OR: 2.46; 95\% CI 1.32-4.57, $P<0.005$ ), Table 4. Moreover, a significant trend between PPI dose and risk of hypomagnesaemia was observed $\left(P_{\text {trend }}=0.004\right)$.

Table 4. Subgroup analyses of the association of PPI use with hypomagnesaemia in 689 kidney transplant recipients.

\begin{tabular}{|c|c|c|c|c|c|c|c|}
\hline & \multicolumn{7}{|c|}{ Categories of PPI Use } \\
\hline & \multicolumn{2}{|c|}{ No PPI } & \multicolumn{2}{|c|}{ Low PPI Dose } & \multicolumn{2}{|c|}{ High PPI Dose } & \\
\hline \multirow[t]{2}{*}{ Number of subjects } & \multicolumn{2}{|l|}{300} & \multicolumn{2}{|c|}{251} & \multicolumn{2}{|c|}{138} & \\
\hline & $\begin{array}{l}\text { Odds ratio } \\
(95 \% \mathrm{CI})\end{array}$ & $P$ & $\begin{array}{c}\text { Odds ratio } \\
(95 \% \mathrm{CI})\end{array}$ & $P$ & $\begin{array}{c}\text { Odds ratio } \\
(95 \% \mathrm{CI})\end{array}$ & $P$ & $P$-Trend \\
\hline \multicolumn{8}{|l|}{ Hypomagnesaemia } \\
\hline Crude & $\begin{array}{c}1.00 \\
\text { (reference) }\end{array}$ & $\mathrm{n} / \mathrm{a}$ & $\begin{array}{c}1.92 \\
(1.25-2.96)\end{array}$ & 0.003 & $\begin{array}{c}2.53 \\
(1.55-4.11)\end{array}$ & $<0.001$ & $<0.001$ \\
\hline Multivariable model & $\begin{array}{c}1.00 \\
\text { (reference) }\end{array}$ & $\mathrm{n} / \mathrm{a}$ & $\begin{array}{c}1.79 \\
(1.04-3.08)\end{array}$ & 0.04 & $\begin{array}{c}2.46 \\
(1.32-4.57)\end{array}$ & 0.005 & 0.004 \\
\hline
\end{tabular}

Multivariable analyses were adjusted for age, sex, BMI, eGFR, proteinuria, time since transplantation, alcohol use, diabetes, history of CV disease, loop diuretics, thiazide diuretics, tacrolimus use, cyclosporine use, MMF use, dietary magnesium intake. Low PPI dose ( $\leq 20 \mathrm{mg}$ omeprazole Eq/day), High PPI dose ( $>20 \mathrm{mg}$ omeprazole Eq/day). Abbreviations: $\mathrm{CI}$, confidence interval.

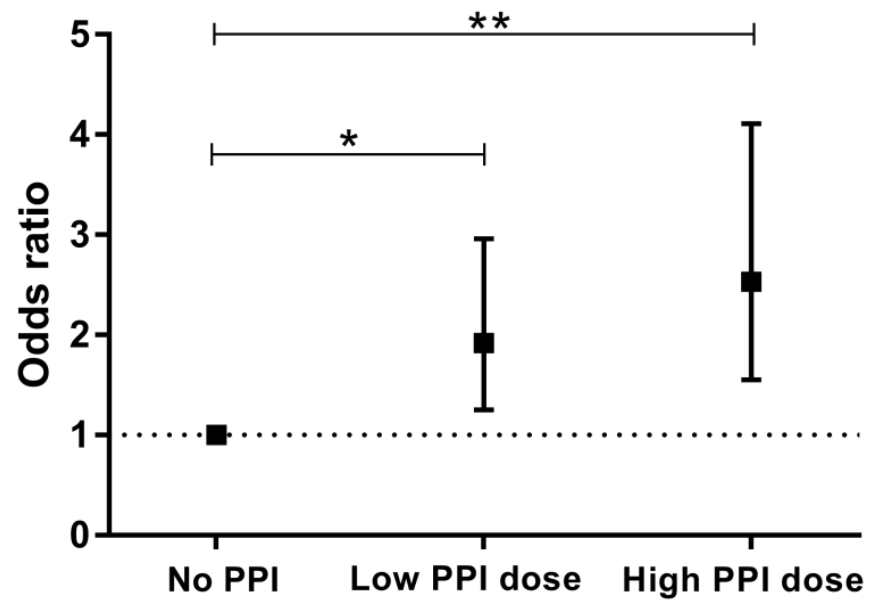

Figure 1. Crude association between PPI use and risk of hypomagnesaemia stratified by subgroups of PPI use. No PPI, Low PPI dose ( $\leq 20 \mathrm{mg}$ omeprazole Eq/day), High PPI dose ( $>20 \mathrm{mg}$ omeprazole Eq/day). Presented are odds ratio's with $95 \%$ confidence intervals. ${ }^{*} P=0.004 ;{ }^{* *} P<0.001 ; P_{\text {trend }}<0.001$. 


\subsection{Sensitivity Analyses for Risk of Hypomagnesaemia}

To account for the use of other important gastric acid reducing medication, we performed sensitivity analyses in which H2RA users $(N=18)$ were excluded form statistical analyses (Table S2). The association between PPI use and hypomagnesaemia remained materially unchanged when H2RA users were excluded (OR: 2.17, 95\% CI 1.29-3.67, $P=0.004$ ). We also performed analyses to investigate which KTR are at increased risk of developing hypomagnesaemia. These analyses are presented in Table S3. We found that patients with a history of cardiovascular disease, patients at shorter time after transplantation, not consuming alcohol, PPI users, thiazide diuretic users and patients using tacrolimus based immunosuppressive regimens were at increased risk of developing hypomagnesaemia. Moreover, KTR with hypomagnesaemia had higher fasting glucose levels, HbA1c and lower serum calcium levels compared with KTR without hypomagnesaemia.

\section{Discussion}

The present study is to our knowledge the largest cohort study to date exploring the association between PPI use and hypomagnesaemia in a cohort of KTR. Our results demonstrate a higher risk of hypomagnesaemia among KTR using PPIs, with subsequently lower plasma magnesium levels in combination with lower renal magnesium excretion. The association between PPI use and risk of hypomagnesaemia remained significant after adjustment for important potential confounders and tended to be highest among KTR taking high PPI dosages.

Our results confirm previous case-series and cohort studies investigating the association between PPI use and increased risk of hypomagnesaemia [1,2,7,9]. In a large cohort study $(N=9818)$ among subjects from the general population, it was shown that PPI users had significantly lower serum magnesium levels and had a two times higher risk of hypomagnesaemia compared with non-users [9]. Our results are in line with observations from this large cohort study and show a similar increased risk of hypomagnesaemia (OR 2.12).

So far, only one other study by van Ende et al. investigating the association between PPI use and magnesium status in KTR has been published [22]. Contrary to our findings, van Ende et al. found no association between PPI use and serum magnesium levels. Reasons for the lower proportion of PPI users in the study by van Ende et al. are unclear, though underreporting may have played a role, given that it was not specified how data regarding PPI use was obtained. It was also unclear whether the data of van Ende et al. were derived from routine outpatient assessment of plasma magnesium concentrations, which may have provided an incentive for stopping PPI use in KTR with low magnesium concentrations. This could have biased their results and could possibly also explain the large difference in PPI use between our study and their study, because in our center no plasma magnesium data were available at the time of the study. It was furthermore unclear whether it concerned on-demand or chronic PPI use. Furthermore, data regarding PPI dose, type and magnesium supplementation were not reported, which may have influenced the outcome.

In a recently published meta-analysis, a similar risk of hypomagnesaemia among KTR was demonstrated (pooled OR $=1.56,95 \%$ CI 1.19-2.05) [30]. This meta-analysis by Boonpheng et al. was based on one published paper and seven abstracts presented at medical conferences. Our study adds that it investigated a dose-response relationship, and provides data on dietary magnesium intake and 24-h urinary magnesium excretion.

In the present study, both plasma magnesium and 24-h urinary magnesium excretion were lower in PPI users, suggesting that PPI induced hypomagnesaemia is caused by impaired gastrointestinal absorption rather than renal magnesium wasting. In general, hypomagnesaemia can be the consequence of either a decreased intestinal uptake, a decrease in dietary magnesium intake or an increase in renal magnesium excretion. It is postulated that PPIs inhibit the active magnesium absorption via the TRPM 6 and 7 channels in the intestine [11,12]. In KTR other contributing factors than PPI use may add to the risk of hypomagnesaemia. For example, decreased intestinal magnesium absorption can also be the consequence of chronic post-transplant diarrhea, which is highly prevalent and 
often complicated by hypomagnesaemia [10,31]. Data regarding symptoms of severe diarrhea were unfortunately unavailable in this study, therefore we could not correct for this potential confounder. Likewise, hypomagnesaemia can be the result of insufficient intake of foods rich in magnesium. In our study, mean dietary magnesium intake was $329.9 \pm 88.7 \mathrm{mg} /$ day, which was slightly lower than the mean habitual intake of magnesium among the general Dutch population, as reported in the Dutch National Food Consumption Survey 2007-2010 [32]. A low dietary magnesium intake can also be a reflection of an overall poor diet. Nonetheless, when we adjusted for dietary magnesium intake in our logistic regression analyses, the relationship between PPI use and risk of hypomagnesaemia remained materially unchanged, indicating that the observed risk associated with PPI use was not confounded by dietary magnesium intake.

The main strength of this study is measurement of three important pillars of magnesium status: plasma magnesium, 24-h urinary magnesium excretion and dietary magnesium intake. Because of this, we were able to confirm that PPI use does not lead to increased renal magnesium wasting but very likely impairs intestinal magnesium absorption. Furthermore, we only included KTR who were using PPIs for at least 3 months before blood sampling. It is previously noted that hypomagnesaemia occurs mainly in patients on prolonged PPI therapy suggesting that it takes time before magnesium stores are meaningfully depleted $[6,7,33]$. Moreover, we excluded KTR using magnesium supplements and adjusted for potential confounders, including CNI use, which did not alter the association.

A limitation of our study is its cross-sectional design. Therefore, a causal relationship between PPI use and hypomagnesaemia remains to be determined and changes over time in magnesium status parameters were unknown. Furthermore, no information regarding compliance to PPI treatment was available, which may have led to underestimation of effect sizes. PPI users had a shorter time between transplantation and baseline measurements. However, adjustment for time since transplantation did not alter the association between PPI use and hypomagnesaemia. Lastly, the possibility of residual confounding or bias by indication remains, which may have led to overestimation of the role of PPIs since on average PPI users were less healthy than non-users. A strength of the current study is, that no routine outpatient monitoring of plasma magnesium was performed and that we measured plasma and urine magnesium in samples that had been stored in a biobank, which reduces the change of selection bias in our cohort.

Our findings may be of clinical importance. KTR with low magnesium levels seem to develop post-transplant diabetes mellitus (PTDM) more frequently [17]. In this study we also found that KTR with hypomagnesaemia had higher fasting glucose levels and HbA1c. Next to that, a higher degree of arterial stiffness, as assessed by a carotid-femoral pulse wave velocity (PWV) measurement, has been found in KTR with low magnesium levels [34]. This same PWV measurement was found to be an independent predictor of cardiovascular events in KTR [35]. Moreover, hypomagnesaemia has been associated with cardiovascular morbidity $[19,20]$ and mortality $[21]$ in the general population. However, whether this association is also present in KTR is currently unknown. Another clinical significance lies in the association with lower calcium levels, which potentially points to an increased risk of developing osteoporosis. Long-term PPI use has indeed been associated with decreased bone mineral density and increased risk of fractures [36]. Because many patients use PPIs without evidence based indication [37-39], we believe that reevaluation of treatment indication in KTR on chronic PPI therapy might be of benefit. In situations in which PPIs are clinically needed, it would be judicious to assess and follow-up magnesium levels periodically during treatment, as recommended by the US Food and Drug administration and stated in the summary of product characteristics of all PPIs.

\section{Conclusions}

This study demonstrates that PPI use is associated with lower magnesium status and hypomagnesaemia in KTR. Moreover, risk of hypomagnesaemia was higher among KTR taking a high PPI dosage. Healthcare professionals should be aware of this additional risk and should 
consider regular monitoring of magnesium levels, especially in this patient population at high risk of hypomagnesaemia.

Supplementary Materials: The following are available online at http://www.mdpi.com/2077-0383/8/12/2162/ s1, Table S1: Types and daily dosages of proton-pump inhibitors used by 389 kidney transplant recipients. Table S2: Logistic regression analyses investigating the association of PPI use with hypomagnesaemia in 617 kidney transplant recipients (H2RA users excluded). Table S3: Baseline characteristics of 689 RTR with and without hypomagnesaemia.

Author Contributions: Data curation, R.M.D., A.W.G.-N., E.v.d.B., and S.J.L.B.; Formal analysis, R.M.D., A.W.G.-N., J.C.S. and S.J.L.B.; Methodology, R.M.D, A.W.G.-N., J.C.S., E.v.d.B., M.H.d.B., S.P.B., D.J.T., E.H., H.B., G.J.N. and S.J.L.B. writing—original draft preparation, R.M.D., A.W.G.-N.; writing—review and editing, R.M.D., A.W.G.-N., J.C.S., M.H.d.B., E.v.d.B., S.P.B., D.J.T., E.H., H.B., G.J.N. and S.J.L.B. Supervision, H.B., G.N., S.J.L.B.; Funding acquisition, R.M.D., E.v.d.B. and S.J.L.B.

Funding: Generation of this study was funded by Top Institute Food and Nutrition (grant A-1003). R.M. Douwes is supported by NWO/TTW in a partnership program with DSM, Animal Nutrition and Health, The Netherlands; grant number: 14939.

Conflicts of Interest: M.H. de Borst is the principal investigator of a clinical trial supported by the Dutch Kidney Foundation (grant no 17OKG18) and Vifor Fresenius Medical Care Renal Pharma. He received consultancy fees (to employer) from Amgen, Astra Zeneca, Bayer, Kyowa Kirin, Sanofi Genzyme, and Vifor Fresenius Medical Care Renal Pharma. D.J. Touw has received grants (to employer) from ZONMw, Astellas and Chiesi Pharmaceuticals for research outside this project. None of these entities had any role in the design, collection, analysis, and interpretation of data for the current study, nor in writing the report or the decision to submit the report for publication. The other authors declare that they have no other relevant financial interests.

\section{References}

1. Cundy, T.; Dissanayake, A. Severe hypomagnesaemia in long-term users of proton-pump inhibitors. Clin. Endocrinol. (Oxf.) 2008, 69, 338-341. [CrossRef] [PubMed]

2. Danziger, J.; William, J.H.; Scott, D.J.; Lee, J.; Lehman, L.W.; Mark, R.G.; Howell, M.D.; Celi, L.A.; Mukamal, K.J. Proton-pump inhibitor use is associated with low serum magnesium concentrations. Kidney Int. 2013, 83, 692-699. [CrossRef] [PubMed]

3. Hess, M.W.; Hoenderop, J.G.; Bindels, R.J.; Drenth, J.P. Systematic review: Hypomagnesaemia induced by proton pump inhibition. Aliment. Pharmacol. Ther. 2012, 36, 405-413. [CrossRef] [PubMed]

4. Park, C.H.; Kim, E.H.; Roh, Y.H.; Kim, H.Y.; Lee, S.K. The association between the use of proton pump inhibitors and the risk of hypomagnesemia: A systematic review and meta-analysis. PLoS ONE 2014, 9, e112558. [CrossRef]

5. Cheungpasitporn, W.; Thongprayoon, C.; Kittanamongkolchai, W.; Srivali, N.; Edmonds, P.J.; Ungprasert, P.; O'Corragain, O.A.; Korpaisarn, S.; Erickson, S.B. Proton pump inhibitors linked to hypomagnesemia: A systematic review and meta-analysis of observational studies. Ren. Fail. 2015, 37, 1237-1241. [CrossRef]

6. Regolisti, G.; Cabassi, A.; Parenti, E.; Maggiore, U.; Fiaccadori, E. Severe hypomagnesemia during long-term treatment with a proton pump inhibitor. Am. J. Kidney Dis. 2010, 56, 168-174. [CrossRef]

7. Hoorn, E.J.; van der Hoek, J.; de Man, R.A.; Kuipers, E.J.; Bolwerk, C.; Zietse, R. A case series of proton pump inhibitor-induced hypomagnesemia. Am. J. Kidney Dis. 2010, 56, 112-116. [CrossRef]

8. Lindner, G.; Funk, G.-C.; Leichtle, A.B.; Fiedler, G.M.; Schwarz, C.; Eleftheriadis, T.; Pasch, A.; Mohaupt, M.G.; Exadaktylos, A.K.; Arampatzis, S. Impact of proton pump inhibitor use on magnesium homoeostasis: A cross-sectional study in a tertiary emergency department. Int. J. Clin. Pract. 2014, 68, 1352-1357. [CrossRef]

9. Kieboom, B.C.; Jong, J.C.K.; Eijgelsheim, M.; Franco, O.H.; Kuipers, E.J.; Hofman, A.; Zietse, R.; Stricker, B.H.; Hoorn, E.J. Proton pump inhibitors and hypomagnesemia in the general population: A population-based cohort study. Am. J. Kidney Dis. 2015, 66, 775-782. [CrossRef]

10. de Baaij, J.H.F.; Hoenderop, J.G.J.; Bindels, R.J.M. Magnesium in Man: Implications for Health and Disease. Physiol. Rev. 2015, 95, 1-46. [CrossRef]

11. William, J.H.; Danziger, J. Proton-pump inhibitor-induced hypomagnesemia: Current research and proposed mechanisms. World J. Nephrol. 2016, 5, 152-157. [CrossRef] [PubMed]

12. Schlingmann, K.P.; Waldegger, S.; Konrad, M.; Chubanov, V.; Gudermann, T. TRPM6 and TRPM7-Gatekeepers of human magnesium metabolism. Biochim. Biophys. Acta Mol. Basis Dis. 2007, 1772, 813-821. [CrossRef] [PubMed] 
13. William, J.H.; Nelson, R.; Hayman, N.; Mukamal, K.J.; Danziger, J. Proton-pump inhibitor use is associated with lower urinary magnesium excretion. Nephrology (Carlton) 2014, 19, 798-801. [CrossRef] [PubMed]

14. Barton, C.H.; Vaziri, N.D.; Martin, D.C.; Choi, S.; Alikhani, S. Hypomagnesemia and renal magnesium wasting in renal transplant recipients receiving cyclosporine. Am. J. Med. 1987, 83, 693-699. [CrossRef]

15. Vannini, S.D.; Mazzola, B.L.; Rodoni, L.; Truttmann, A.C.; Wermuth, B.; Bianchetti, M.G.; Ferrari, P. Permanently reduced plasma ionized magnesium among renal transplant recipients on cyclosporine. Transpl. Int. 1999, 12, 244-249. [CrossRef]

16. Van de Cauter, J.; Sennesael, J.; Haentjens, P. Long-term evolution of the mineral metabolism after renal transplantation: A prospective, single-center cohort study. Transplant. Proc. 2011, 43, 3470-3475. [CrossRef]

17. Van Laecke, S.; Van Biesen, W.; Verbeke, F.; De Bacquer, D.; Peeters, P.; Vanholder, R. Posttransplantation hypomagnesemia and its relation with immunosuppression as predictors of new-onset diabetes after transplantation. Am. J. Transplant. 2009, 9, 2140-2149. [CrossRef]

18. Augusto, J.-F.; Subra, J.-F.; Duveau, A.; Rakotonjanahary, J.; Dussaussoy, C.; Picquet, J.; Croue, A.; Villemain, F.; Onno, C.; Sayegh, J. Relation between pretransplant magnesemia and the risk of new onset diabetes after transplantation within the first year of kidney transplantation. Transplantation 2014, 97, 1155-1160. [CrossRef]

19. Joosten, M.M.; Gansevoort, R.T.; Mukamal, K.J.; van der Harst, P.; Geleijnse, J.M.; Feskens, E.J.; Navis, G.; Bakker, S.J.; Group, P.S. Urinary and plasma magnesium and risk of ischemic heart disease. Am. J. Clin. Nutr. 2013, 97, 1299-1306. [CrossRef]

20. Joosten, M.M.; Gansevoort, R.T.; Mukamal, K.J.; Kootstra-Ros, J.E.; Feskens, E.J.; Geleijnse, J.M.; Navis, G.; Bakker, S.J.; Group, P.S. Urinary magnesium excretion and risk of hypertension: The prevention of renal and vascular end-stage disease study. Hypertension 2013, 61, 1161-1167. [CrossRef]

21. Xu, T.; Sun, Y.; Xu, T.; Zhang, Y. Magnesium intake and cardiovascular disease mortality: A meta-analysis of prospective cohort studies. Int. J. Cardiol. 2013, 167, 3044-3047. [CrossRef] [PubMed]

22. Van Ende, C.; Van Laecke, S.; Marechal, C.; Verbeke, F.; Kanaan, N.; Goffin, E.; Vanholder, R.; Jadoul, M. Proton-pump inhibitors do not influence serum magnesium levels in renal transplant recipients. J. Nephrol. 2014, 27, 707-711. [CrossRef] [PubMed]

23. van den Berg, E.; Engberink, M.F.; Brink, E.J.; van Baak, M.A.; Joosten, M.M.; Gans, R.O.; Navis, G.; Bakker, S.J. Dietary acid load and metabolic acidosis in renal transplant recipients. Clin. J. Am. Soc. Nephrol. 2012, 7, 1811-1818. [CrossRef] [PubMed]

24. Douwes, R.M.; Gomes-Neto, A.W.; Eisenga, M.F.; Vinke, J.S.J.; de Borst, M.H.; van den Berg, E.; Berger, S.P.; Touw, D.J.; Hak, E.; Blokzijl, H.; et al. Chronic Use of Proton-Pump Inhibitors and Iron Status in Renal Transplant Recipients. J. Clin. Med. 2019, 8, 1382. [CrossRef]

25. Kirchheiner, J.; Glatt, S.; Fuhr, U.; Klotz, U.; Meineke, I.; Seufferlein, T.; Brockmoller, J. Relative potency of proton-pump inhibitors-comparison of effects on intragastric pH. Eur. J. Clin. Pharmacol. 2009, 65, $19-31$. [CrossRef]

26. van den Berg, E.; Engberink, M.F.; Brink, E.J.; van Baak, M.A.; Gans, R.O.B.; Navis, G.; Bakker, S.J.L. Dietary protein, blood pressure and renal function in renal transplant recipients. Br. J. Nutr. 2013, 109, 1463-1470. [CrossRef]

27. Feunekes, I.J.; Van Staveren, W.A.; Graveland, F.; De Vos, J.; Burema, J. Reproducibility of a semiquantitative food frequency questionnaire to assess the intake of fats and cholesterol in The Netherlands. Int. J. Food Sci. Nutr. 1995, 46, 117-123. [CrossRef]

28. The Netherlands Nutrition Centre. Dutch Food Composition Table 2006 NEVO-Tabel: Nederlands Voedingsstoffenbestand; The Netherlands Nutrition Centre: The Hague, The Netherlands, 2006.

29. van den Berg, E.; Geleijnse, J.M.; Brink, E.J.; van Baak, M.A.; van der Heide, J.J.H.; Gans, R.O.; Navis, G.; Bakker, S.J. Sodium intake and blood pressure in renal transplant recipients. Nephrol. Dial. Transplant. 2012, 27, 3352-3359. [CrossRef]

30. Boonpheng, B.; Thongprayoon, C.; Bathini, T.; Sharma, K.; Mao, M.A.; Cheungpasitporn, W. Proton pump inhibitors and adverse effects in kidney transplant recipients: A meta-analysis. World J. Transplant. 2019, 9, 35-47. [CrossRef]

31. Aulagnon, F.; Scemla, A.; DeWolf, S.; Legendre, C.; Zuber, J. Diarrhea After Kidney Transplantation. Transplantation 2014, 98, 806-816. [CrossRef] 
32. van Rossum, C.T.M.; Fransen, H.; Verkaik-Kloosterman, J.; Buurma-Rethans, E.; Ocke, M. Micronutrients. In Dutch National Food Consumption Survey 2007-2010: Diet of Children and Adults Aged 7 to 69 Years; National Institute for Public Health and the Environment (RIVM): Bilthoven, The Netherlands, 2011; pp. 61-76.

33. Bai, J.P.F.; Hausman, E.; Lionberger, R.; Zhang, X. Modeling and simulation of the effect of proton pump inhibitors on magnesium homeostasis. 1. Oral absorption of magnesium. Mol. Pharm. 2012, 9, 3495-3505. [CrossRef] [PubMed]

34. Van Laecke, S.; Maréchal, C.; Verbeke, F.; Peeters, P.; Van Biesen, W.; Devuyst, O.; Jadoul, M.; Vanholder, R. The relation between hypomagnesaemia and vascular stiffness in renal transplant recipients. Nephrol. Dial. Transplant. 2011, 26, 2362-2369. [CrossRef] [PubMed]

35. Verbeke, F.; Maréchal, C.; Van Laecke, S.; Van Biesen, W.; Devuyst, O.; Van Bortel, L.M.; Jadoul, M.; Vanholder, R. Aortic stiffness and central wave reflections predict outcome in renal transplant recipients. Hypertension 2011, 58, 833-838. [CrossRef] [PubMed]

36. Thong, B.K.S.; Ima-Nirwana, S.; Chin, K.Y. Proton pump inhibitors and fracture risk: A review of current evidence and mechanisms involved. Int. J. Environ. Res. Public Health 2019, 16, 1571. [CrossRef]

37. Zink, D.A.; Pohlman, M.; Barnes, M.; Cannon, M.E. Long-term use of acid suppression started inappropriately during hospitalization. Aliment. Pharmacol. Ther. 2005, 21, 1203-1209. [CrossRef]

38. Batuwitage, B.T.; Kingham, J.G.; Morgan, N.E.; Bartlett, R.L. Inappropriate prescribing of proton pump inhibitors in primary care. Postgrad. Med. J. 2007, 83, 66-68. [CrossRef]

39. Nardino, R.J.; Vender, R.J.; Herbert, P.N. Overuse of acid-suppressive therapy in hospitalized patients. Am. J. Gastroenterol. 2000, 95, 3118-3122. [CrossRef]

(C) 2019 by the authors. Licensee MDPI, Basel, Switzerland. This article is an open access article distributed under the terms and conditions of the Creative Commons Attribution (CC BY) license (http://creativecommons.org/licenses/by/4.0/). 